\title{
Synthesis of acridone derivatives via heterologous expression of a plant type III polyketide synthase in Escherichia coli
}

\author{
Gyu-Sik Choi ${ }^{1}$, Hye Jeong Choo ${ }^{1}$, Bong-Gyu Kim² and Joong-Hoon Ahn ${ }^{1 *}$ (D)
}

\begin{abstract}
Background: Acridone alkaloids are heterocyclic compounds that exhibit a broad-range of pharmaceutical and chemotherapeutic activities, including anticancer, antiviral, anti-inflammatory, antimalarial, and antimicrobial effects. Certain plant species such as Citrus microcarpa, Ruta graveolens, and Toddaliopsis bremekampii synthesize acridone alkaloids from anthranilate and malonyl-CoA.
\end{abstract}

Results: We synthesized two acridones in Escherichia coli. Acridone synthase (ACS) and anthraniloyl-CoA ligase genes were transformed into E. coli, and the synthesis of acridone was examined. To increase the levels of endogenous anthranilate, we tested several constructs expressing proteins involved in the shikimate pathway and selected the best construct. To boost the supply of malonyl-CoA, genes coding for acetyl-coenzyme A carboxylase (ACC) from Photorhabdus luminescens were overexpressed in E. coli. For the synthesis of 1,3-dihydroxy-10-methylacridone, we utilized an N-methyltransferase gene (NMT) to supply N-methylanthranilate and a new N-methylanthraniloyl-CoA ligase. After selecting the best combination of genes, approximately $17.3 \mathrm{mg} / \mathrm{L}$ of 1,3-dihydroxy-9(10H)-acridone (DHA) and $26.0 \mathrm{mg} / \mathrm{L}$ of 1,3-dihydroxy-10-methylacridone (NMA) were synthesized.

Conclusions: Two bioactive acridone derivatives were synthesized by expressing type III plant polyketide synthases and other genes in E. coli, which increased the supplement of substrates. This study showed that is possible to synthesize diverse polyketides in E. coli using plant polyketide synthases.

Keywords: Acridone, Metabolic engineering, Polyketide synthase

\section{Background}

Natural compounds are valuable in cosmetics, food, and pharmaceutical industries [1]. Therefore, natural and nature-inspired, chemically synthesized compounds have extensively been developed and exploited for countless industrial purposes. Phytochemicals are typical natural compounds that have additional biological, nutritive, and/or pharmacological value. Among the diverse phytochemicals, secondary metabolites such as alkaloids,

*Correspondence: jhahn@konkuk.ac.kr

${ }^{1}$ Department of Bioscience and Biotechnology, Bio/Molecular Informatics Center, Konkuk University, Seoul 05029, Republic of Korea

Full list of author information is available at the end of the article phenylpropanoids, and terpenoids have been extensively studied, and some of them have been employed in various fields [2].

Acridones are heterocyclic alkaloids that contain a tricyclic ring with nitrogen at the 10th position and a carbonyl group at the 9th position [3]. Acridone alkaloids are secondary metabolites that are generally found in the plant family, Rutaceae [4]. Various acridone derivatives (glyforine, acronycine, thioacridones, and substituted 9-aminoacridines, etc.) have been reported to exert a wide range of chemotherapeutic effects including anticancer, antimicrobial, antimalarial, antipsoriatic activities [5-8]. The synthesis of acridone alkaloids in plants 
(Rutaceae family) was reported several decades after the discovery of acridine as a derivative of coal tar [9].

$N$-methylacridone (1,3-dihydroxy-10-methylacridone) was discovered in Ruta graveolens [10]. Some genes in acridone biosynthesis have been characterized, and the acridone biosynthetic pathway has been elucidated in $R$. graveolens. The first committed step in acridone alkaloid biosynthesis is the conversion of anthranilate into $N$-methylanthranilate by anthranilate $N$-methyltransferase (ANMT). Next is the $N$-methylanthranilatecatalyzed synthesis of $\mathrm{N}$-methylanthraniloyl-CoA using coenzyme A (CoA). Then, acridone synthase (ACS) - one of the plant polyketide synthases (PKSs)-catalyzes the condensation of $N$-methylanthraniloyl-CoA and malonylCoA. ANMT and ACS were cloned in R. graveolens [11, 12]. ACSs from Huperzia serrata [13] and Citrus microcarpa [14] have also been cloned. Notably, to date, plant anthranilate coenzyme A ligase has not been cloned.

Microorganic biosynthetic platforms have emerged as the leading platforms for the production of natural and synthetic value-added compounds, such as flavonoids, alkaloids, polyketides, and various chemicals. Due to its well-established genetics and physiology, Escherichia coli has become one of the representative microorganisms in biosynthetic platforms [15]. One of the secondary metabolic pathways of $E$. coli, the shikimate pathway has received considerable attention as it is a major pathway for the production of aromatic compounds [16]. Biosynthetic pathways for aromatic amino acid production (L-tryptophan, L-tyrosine and L-phenylalanine) including the shikimic acid pathway provide the chemical building blocks for the synthesis of various chemicals through specific intermediates, such as chorismate and shikimate [17-21].

We synthesized two acridones (1,3-dihydroxy-9(10H)acridone [DHA] and 1,3-dihydroxy-10-methylacridone [NMA]) using engineered E. coli and two substrates, namely anthranilate, and malonyl-CoA. To optimize the substrate supply for the synthesis of acridone, we prepared several sets of constructs; the first set for the synthesis of anthranilate using genes coding for proteins involved in the shikimate pathway and the second set for the synthesis of malonyl-CoA by overexpressing acetylcoenzyme A carboxylases (ACCs). For the synthesis of NMA (1,3-dihydroxy-10-methylacridone), we additionally introduced the $N$-methyltransferase gene (NMT) to supply $N$-methylanthranilate by using endogenous anthranilate. The overall scheme of the biosynthesis of these two compounds is shown in Fig. 1. Through a combination of these genes along with $A C S, b a d A$, and $p q s A$, which are involved in CoA utilization or substrate cyclization, we were able to synthesize $17.3 \mathrm{mg} / \mathrm{L} \mathrm{DHA}$ and $26.0 \mathrm{mg} / \mathrm{L}$ NMA.

\section{Results}

Screening of constructs to synthesize DHA and NMA

DHA and NMA are synthesized from anthranilate or $N$-methylanthranilate and malonyl-CoA, respectively. Anthranilate and $N$-methylanthranilate are activated by coenzyme A. We tested two CoA ligases, badA-encoding benzoate coenzyme A ligase-and $p q s A$ encoding anthranilate coenzyme A ligase. Two ACSs, RgACS, and CmACS were tested. E. coli-harboring each of the four constructs pC-RgACS-badA, pC-CmACS-badA, RgACSpqsA or pC-CmACS-pqsA-was exposed to $100 \mu \mathrm{M}$ anthranilate or $N$-methylanthranilate. A new peak was observed in culture filtrates from $E$. coli strains harboring RgACS-badA or pC-CmACS-badA when they were supplied with anthranilate (Fig. 2d, e). The molecular mass of the synthesized product was 227.06 Da, which corresponded to the predicted mass of DHA. However, E. coli cells harboring RgACS-pqsA or pC-CmACS-pqsA that were supplied with $N$-methylantrhanilate synthesized a new product whose molecular mass was $240.87 \mathrm{Da}$, which is the predicted mass of NMA (Fig. 2e, g). Based on the structure-using nuclear magnetic resonance spectroscopy (NMR) - we confirmed that the two compounds were DHA and NMA, respectively, (see "Methods"). These results indicated that badA could potentially convert anthranilate into anthraniloyl-CoA and that pqsA is responsible for the conversion of $N$-methylanthranilate into $N$-methylanthraniloyl-CoA.

Escherichia coli strains harboring $\operatorname{RgACS}$ synthesized $11.80 \mathrm{mg} / \mathrm{L}$ DHA $(51.96 \mu \mathrm{M})$ when $100 \mu \mathrm{M}$ anthranilate was supplied, and synthesized $17.52 \mathrm{mg} / \mathrm{L}(72.62 \mu \mathrm{M})$ NMA when $100 \mu \mathrm{M} N$-methylanthranilate was provided. This yield exceeded that obtained using $E$. coli harboring $C m A C S$, which synthesized $1.4 \mathrm{mg} / \mathrm{L}$ DHA and $6.0 \mathrm{mg} / \mathrm{L}$ NMA. In addition, the amount of byproduct such as 2,3-dihydroxyquinoline (DHQ) were found more in $E$. coli harboring $C m A C S$ and the unreacted $N$-methylanthranilate was observed in $E$. coli harboring $C m A C S$. This result indicates that RgACS effectively synthesizes DHA and NMA. We observed the synthesis of 2,4-dihydroxyquinline (DHQ) in E. coli strains harboring RgACS-badA or CmACS-badA. DHQ also used anthranoyl-CoA and malonyl-CoA. Two molecules of malonyl-CoA instead of three, are used to synthesize DHQ. The amount of the synthesized DHQ was $2.6 \mathrm{mg} / \mathrm{L}$ in $E$. coli harboring CmACS-badA and $3.6 \mathrm{mg} / \mathrm{L}$ in $E$. coli harboring RgACS-badA, while the amount of DHA was $1.3 \mathrm{mg} / \mathrm{L}$ in CmACS and $10.5 \mathrm{mg} / \mathrm{L}$ in RgACS.

The synthesis of $N$-methylquinoline (NMQ) was observed in the culture filtrate of $E$. coli harboring CmACS-pqsA. Nevertheless, we could not observe any detectable NMQ in E. coli harboring RgACS-pqsA. Enzymatic reactions with $N$-methylanthranilate using 


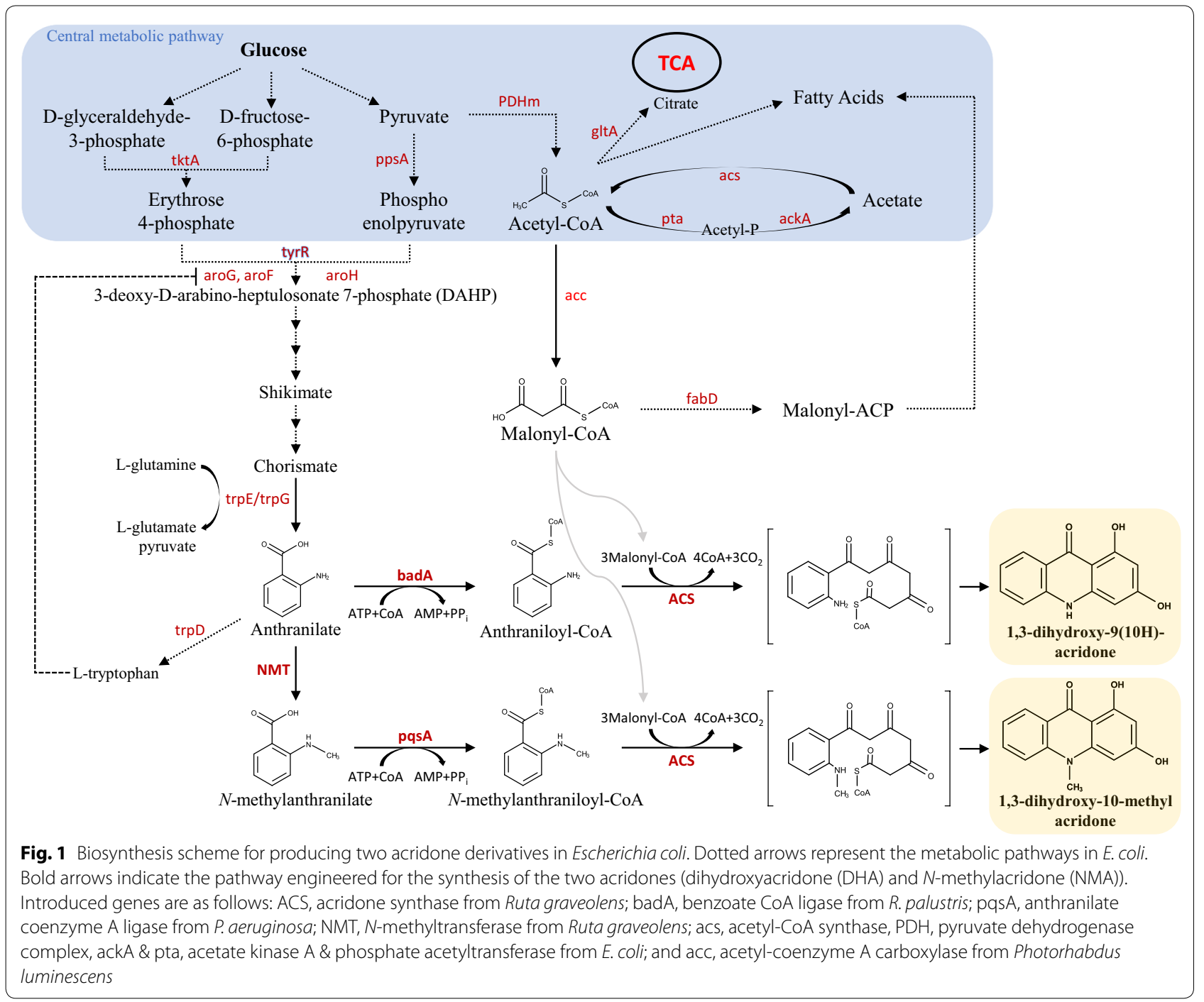

CmANS revealed that the synthesized products resulted from the incorporation of two ( $N$-methylquinolone) or three molecules ( $N$-methylacridone) of malonyl-CoA with a preference towards $N$-methylacridone synthesis [14]. However, the enzymatic reaction using RgACS with $N$-methylanthranilate produced only NMA (but not $\mathrm{N}$-methylquinolone) [22]. These results indicate that RgACS is better than CmACS at synthesizing DHA and NMA. Therefore, we selected constructs containing RgACS for further experiments.

\section{Synthesis of NMA}

$N$-methylanthranilate is the building block of NMA, but $E$. coli does not synthesize $N$-methylanthranilate. Anthranilate NMT was employed to synthesize NMA. In order to increase the substrate for NMT, $\operatorname{trpE}$ was overexpressed. The second substrate of NMA synthesis is malonyl-CoA. The effects the four constructs that reportedly affect intracellular malonyl-CoA were individually tested with respect to NMA synthesis. Three of them (PDHm, acs, and ackA-pta) increased the level of acetyl-CoA [23, 24] and one of them (acc) synthesized malonyl-CoA from acetyl-CoA [24]. We engineered five E. coli strains (B-NMA3-B-NMA7) and tested the synthesis of NMA. Four strains synthesized NMA. Among them, the strain B-NMA3 produced the highest amount of NMA (30.6 mg/L) followed by B-NMA4 $(24.2 \mathrm{mg} / \mathrm{L})$, B-NMA5 $(22.2 \mathrm{mg} / \mathrm{L}), \quad B-N M A 6 \quad(19.3 \mathrm{mg} / \mathrm{L})$, and B-NMA7 (18.3 mg/L) (Fig. 3). The E. coli strains harboring pE-RgACS-PqsA, pC-NMT-TrpE, and the empty pA vector synthesized approximately $18.3 \mathrm{mg} / \mathrm{L}$ NMA. The overexpression of acc increased NMA synthesis $(\sim 1.7-$ fold), followed by pta-ackA ( 1.3-fold), PDHm ( 1.2fold), and acs ( 1.1-fold). These results indicated that 


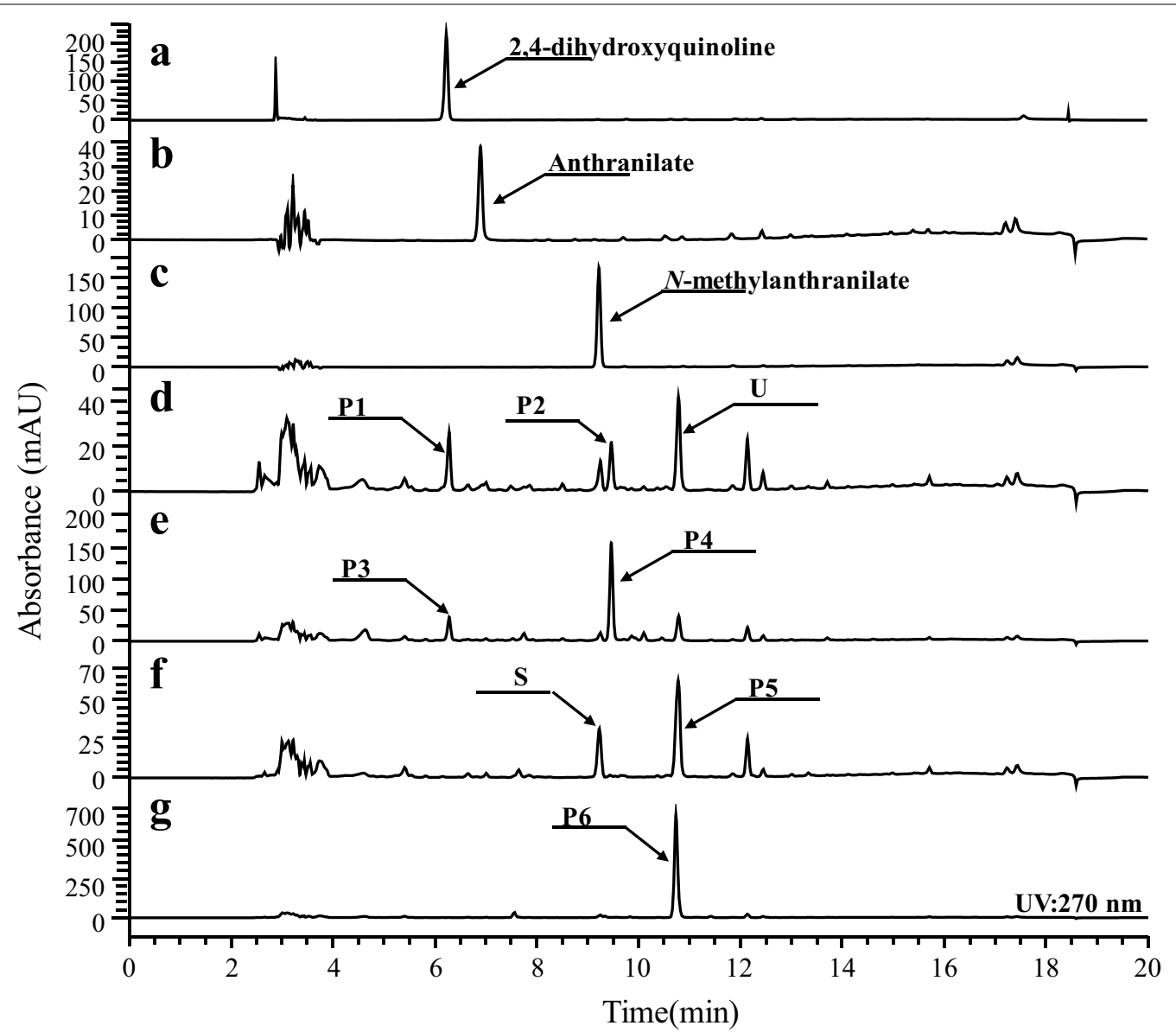

Fig. 2 Screening of PKS and coenzyme ligase for the in vivo synthesis of DHA and NMA. a Authentic 2,3-dihydroxyquinoline (DHQ); b authentic anthranilate; $\mathbf{c}$ authenic $\mathrm{N}$-methylanthranilate; $\mathbf{d}$ products from $\mathrm{E}$. coli harboring pC-CmACS-BadA; e products from E. coli harboring pC-RgACS-BadA; f products from E. coli harboring pC-CmACS-pqsA; $\mathbf{g}$ products from E. coli harboring pC-RgACS-pqsA. E. coli harboring pC-CmACS-BadA (d), pC-RgACS-BadA (e), pC-CmACS-pqsA (f) or pC-RgACS-pqsA (g) were supplied with $100 \mu \mathrm{M}$ anthranilate (d, e) or $\mathrm{N}$-methylanthranilate (f, $\mathbf{g})$. P1 and P3 were DHQ. P2 and P4 were determined to be DHA by NMR. S was unreacted N-methylanthranilate. P5 and P6 were determined to be NMA by NMR. $U$ was unidentified product which seemed to be an intermediate of DHA and its retention time was slightly different from that of NMA

the overexpression of gene involved in acetyl-CoA or malonyl-CoA increased the synthesis of NMA and the enhancement of malonyl-CoA synthesis by acc is more effective in the synthesis of NMA than the increase of acetyl-CoA by pta-ackA, PDHm, or acs.

We also tried to increase endogenous anthranilate levels by overexpressing aro $G$ and the feedback-inhibitionfree version of aroG (aro $\left.G^{f}\right)$. Two more E. coli strains (B-NMA-8 and B-NMA-9) were tested. However, we could not detect the synthesis of NMA. Only the accumulation of anthranilate and $N$-methylanthranilate was observed. The unreacted anthranilate and $N$-methylanthranilate in B-NMA-8 were 16.0 and $35.0 \mathrm{mg} / \mathrm{L}$, respectively; only $7.2 \mathrm{mg} / \mathrm{L} N$-methylanthranilate was observed in B-NMA-3, whereas anthranilate was not observed.
The rapid synthesis of anthranilate or $N$-methylanthranilate seemingly inhibited the synthesis of NMA. Notably, higher copy number plasmids containing RgACS and pqsA did not further increase NMA synthesis. Likely, the activities of these two downstream proteins got saturated when converting the synthesized $N$-methylanthranilate into NMA. Fine-tuning of the whole process is critical to increasing the final yield of the product $[25,26]$.

Using the strain B-NMA3, we monitored the synthesis of NMA and $N$-methylanthranilate for $27 \mathrm{~h}$. The synthesis of both NMA and $N$-methylanthranilate showed a similar pattern (Fig. 4). Both reached their maximal synthesis at $24 \mathrm{~h}$, at which time, approximately $26.0 \mathrm{mg} / \mathrm{L}$ NMA and $5.4 \mathrm{mg} / \mathrm{L} N$-methylanthranilate were synthesized. 


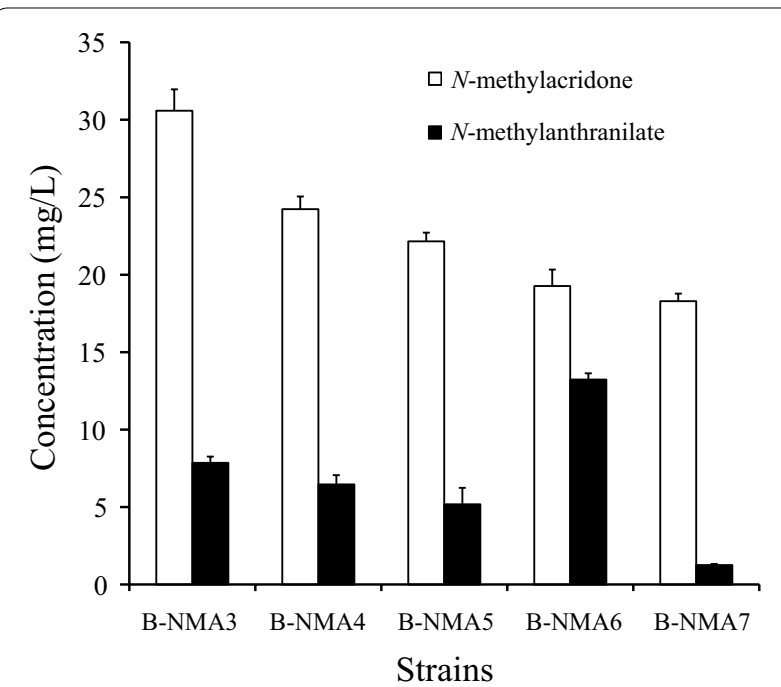

Fig. 3 Effect of the precursor pathway genes on the production of $\mathrm{N}$-methylacridone. B-NMA3 overexpressed acc (acetyl-CoA carboxylase), B-NMA4 overexpressed ackA (acetate kinase), and pta (phosphate acetyltransferase), B-NMA5 overexpressed PDHm (pyruvate dehydrogenase complex variant), and B-NMA6 overexpressed acs (acetyl-coenzyme A synthetase)

\section{Synthesis of DHA}

Anthranilate and malonyl-CoA are substrates for DHA. Endogenous levels of these two compounds are probably critical determinants of the final yield. To increase DHA synthesis, we used two strategies. The first strategy was to increase endogenous anthranilate. The shikimate pathway synthesizes anthranilate. Genes in this pathway were overexpressed. The second strategy was to use a plasmid with different copy number to express RgACS and badA. We constructed eight different $E$. coli strains. The levels of synthesized DHA increased from 2.56 in B-DHA3 to $6.39 \mathrm{mg} / \mathrm{L}$ in D-BHA5, and the strain D-BHA6 produced approximately $3.98 \mathrm{mg} / \mathrm{L}$ of DHA. Importantly, the levels of unreacted anthranilate continued to increase, and were $0.72 \mathrm{mg} / \mathrm{L}$ in B-DHA3 and $593.40 \mathrm{mg} / \mathrm{L}$ in B-DHA6. It seemed that higher production of anthranilate inhibited the synthesis of DHA, and that the conversion of the synthesized anthranilate into DHA was critical for increasing the yield of DHA. In order to augment the conversion of anthranilate, greater and better involvement of downstream genes ( $b a d A$ and $\operatorname{RgACS}$ ) seems necessary. Therefore, we tested the strain B-DHA7-10. The synthesis of DHA increased from $1.12 \mathrm{mg} / \mathrm{L}$ in B-DHA7 to $17.3 \mathrm{mg} / \mathrm{L}$ in B-DHA10 (Fig. 5). In particular, the strain(s) that were expected to synthesize more anthranilate produced more DHA. Besides, the levels of unreacted anthranilate in these strains were less than those in corresponding strains harboring a lower copy of badA and $\operatorname{RgACS}$. Taken together, the higher copy number of $R g A C S$ and badA facilitated the synthesis of DHA.

We also tested the four constructs that were supposed to increase intracellular levels of malonyl-CoA.

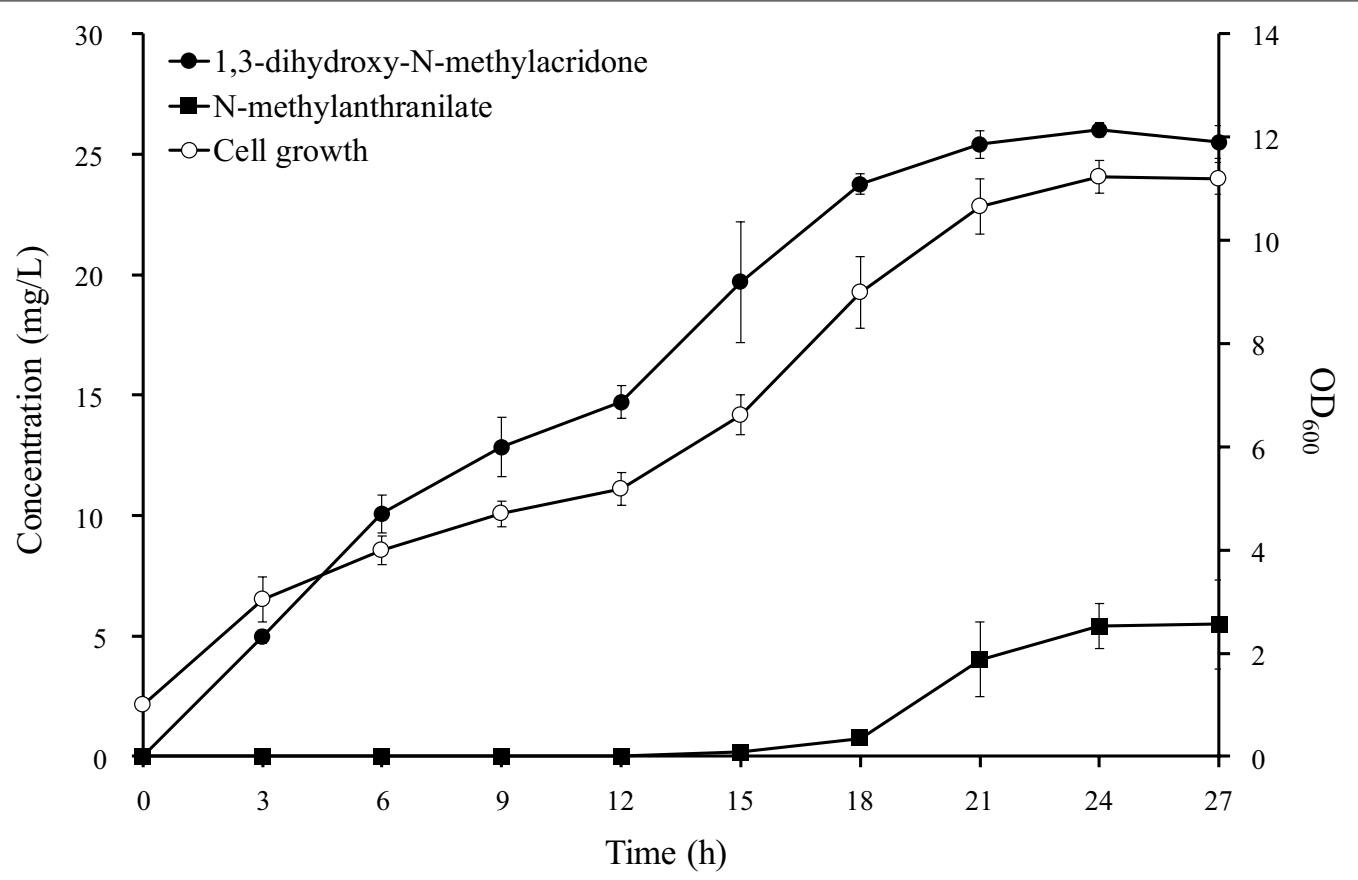

Fig. 4 Production of N-methylacridone using the strain B-NMA3 

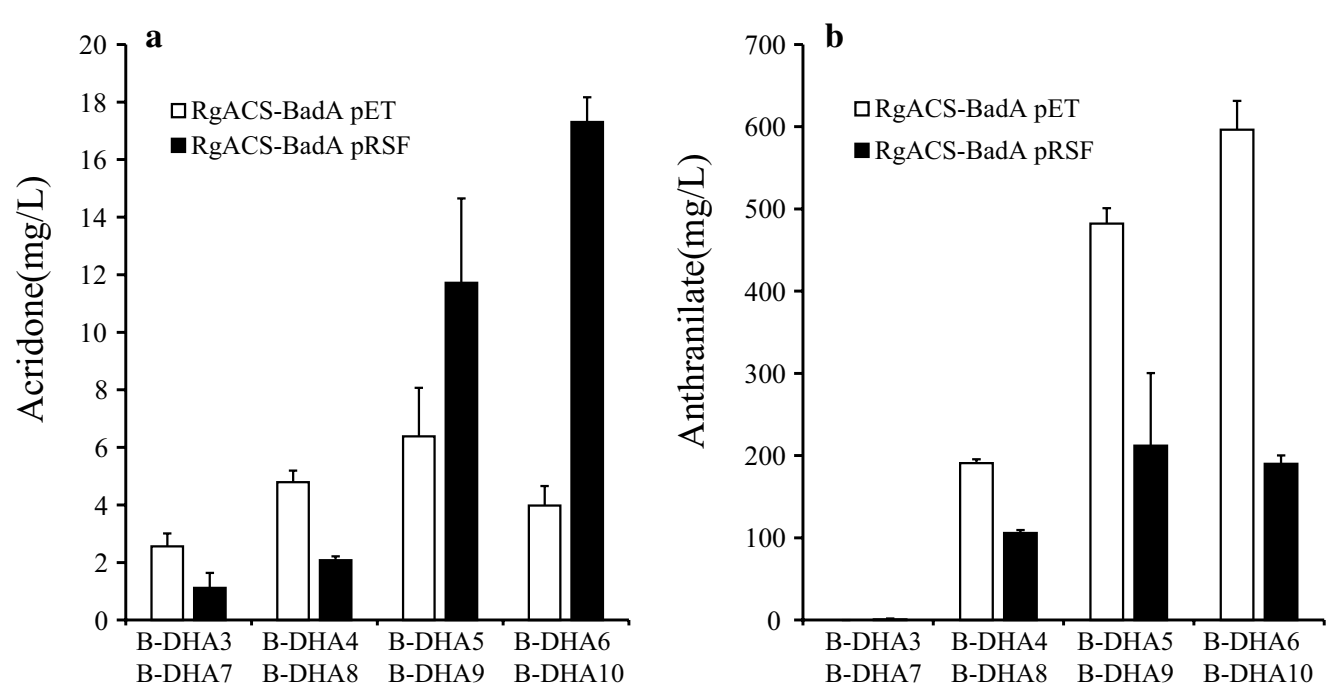

Fig. 5 Optimization of the precursor and vector copy number for acridone production. $\mathbf{a}$ Production of acridone; $\mathbf{b}$ production of anthranilate

Like for the synthesis of NMA, the placcABCD was also the best in the context of DHA synthesis (data not shown). The synthesis of DHA in the strain B-DHA10 was monitored (13 mL of $1 \%$ YM9 broth in $100 \mathrm{~mL}$ flask). DHA levels continued to increase until $15 \mathrm{~h}$ and remained almost the same until $21 \mathrm{~h}$. The levels of unreacted anthranilate also continued to increase until
$40 \mathrm{~h}$. Approximately $15.7 \mathrm{mg} / \mathrm{L}$ DHA was synthesized at $24 \mathrm{~h}$ (Fig. 6).

\section{Discussion}

In our present works, we successfully synthesized two acridone derivatives, 1,3-dihydroxy-9(10H)-acridone and 1,3-dihydroxy-10-methylacridone, using engineered $E$.

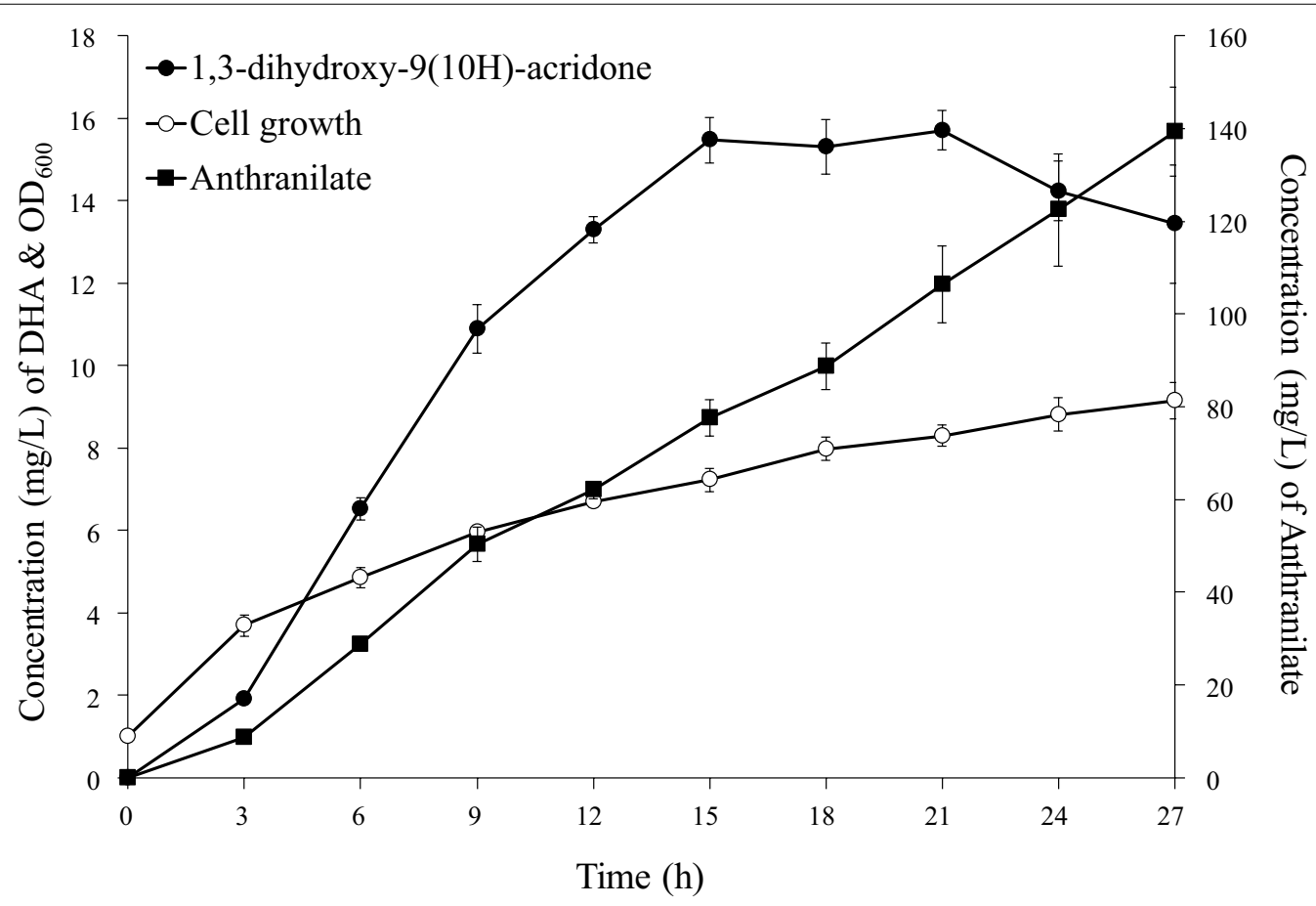

Fig. 6 Production of dihydroxyacridone using the strain B-DHA10 
coli. Genes coding for proteins in the shikimate pathway and $\operatorname{Tr} p E$ encoding anthranilate synthase were tested and selected for the synthesis of the 1st substrate, anthranilate. Acetyl-CoA-carboxylase from $P$. luminescens was introduced to increase the available levels of the 2nd substrate, malonyl-CoA (for ACS). We tested ACS from $R$. graveolens and $C$. microcarpa to select the one that was better with respect to the synthesis of DHA and NMA. The results of in vitro enzymatic efficacy tests showed that ACS from $R$. graveolens outperformed that from $C$. microcarpa [11, 14, 22]. Sometimes, in vitro enzymatic results did not correlate with the in vivo results due to the presence of unknown substrates in vivo, which expectedly inhibit or divert the enzymatic activity [27]. Therefore, we tested the in vivo synthesis of acridone using both genes. In this study, in vivo biosynthesis of acridones by RgACS showed better productivity than CmACS. Based on the in vitro properties of ACS and on the in vivo acridone biosynthesis experiment, we could identify a positive correlation between enzyme properties and acridone biosynthesis.

In order to increase the final yield of the two acridones, we tested the genes coding for proteins involved in the shikimate pathway. We observed a dramatic increase in the levels of intermediates, such as anthranilate, instead of an increase in DHA levels during DHA synthesis. Importantly, during the synthesis of DHA, the rate-limiting step was likely the conversion of anthraniloyl-CoA into DHA by PKS. However, the conversion of anthranilate into $N$-methylanthranilate and/or the conversion of $N$-methylanthraniloyl-CoA into NMA were limiting steps during the synthesis of NMA. Exposure of E. coli harboring CoA ligase and PKS to either anthranilate or $\mathrm{N}$-methylanthranilate resulted in no further synthesis of DHA and NMA, $(\sim 500 \mu \mathrm{M}$ of anthranilate and $300 \mu \mathrm{M}$ of $N$-methylanthranilate). The endogenous levels of anthranilate upon expressing the genes coding for proteins in the shikimate pathway increased more than $500 \mu \mathrm{M}$ (Fig. 5b), a concentration at which the synthesis of DHA is likely inhibited. These findings indicated that PKS was probably a rate-limiting step. In case of NMA synthesis, we found that the conversion of anthranilate into $N$-methylanthranilate was a limiting step [21]. In vitro enzymatic study using the purified ACS from $R$. graveolens also showed that ACS was inhibited by $250 \mu \mathrm{M} \mathrm{N}$-methylanthraniloyl-CoA [28] The construct that minimizes the accumulation of the anthranilate appeared to be the best for the synthesis of NMA and DHA. Fine-tuning of the overall pathway is critical to enhancing the final yield of the product.

Aerobic growth of E. coli produces ATP, ubiquinol-8, $\mathrm{CO}_{2}$ and a considerable amount of acetic acid as a byproduct through the acetate producing pathways [29]. The production of acetic acid could be a negative influence on the synthesis of acridone derivatives. The synthesized acetic acid is neutralized upon converting acetic acid into acetyl-CoA by acetyl-CoA synthase (acs) [30]. Acetyl-CoA is then converted into malonyl-CoA by ACC. Overexpression of acs or acc enhanced the production of DHA and NMA because it resulted not only in the supply of the second substrate (malonyl-CoA), but also in the reduction of the byproduct, acetic acid. These results agreed with the previous reports that overexpression of acs, acc, or $P D H$ enhanced the synthesis of flavonoids and triacetic acid lactone [23, 24, 31].

\section{Conclusions}

We synthesized two acridones (DHA and NMA) in Escherichia coli using two substrates, namely anthranilate and malonyl-CoA. Towards this, plant acridone synthase $(A C S)$ and anthraniloyl-CoA ligase genes were transformed into E. coli. To optimize the substrate supply for acridone synthesis, we prepared several sets of constructs; the first set for the synthesis of anthranilate using genes coding for proteins involved in the shikimate pathway-major pathway for the production of aromatic compounds-and the second set for the synthesis of malonyl-CoA by overexpressing acetyl-coenzyme A carboxylases $(A C C s)$. For the synthesis of NMA, we additionally introduced the $N$-methyltransferase gene (NMT) to supply $N$-methylanthranilate by using endogenous anthranilate. Through a combination of these genes along with $A C S$, badA and $p q s A$, which are involved in CoA utilization or substrate cyclization, we were able to synthesize $17.3 \mathrm{mg} / \mathrm{L}$ DHA and $26.0 \mathrm{mg} / \mathrm{L}$ NMA.

\section{Methods \\ Constructs}

ACS from R. graveolens (RgACS; GenBank: AJ297786.2) was cloned using reverse transcription and polymerase chain reaction (RT-PCR). Two primers $5^{\prime}$-ATGGATCC GATGGAATCCCTGAAGGAGATG- $3^{\prime}$ and $5^{\prime}$-ATGCG GCCGCTCATGCTTCAACGGGGACAC- $3^{\prime}$ were used (restriction sites for EcoRI and NotI have been underlined). ACS from Citrus microcarpa (GenBank: AB823699) was also cloned by RT-PCR using two primers $5^{\prime}$-aagaattcaATGGTAACCATGGAGGAGATTA- $3^{\prime}$ and $5^{\prime}$-aagcggccgcTCATGCTTCTATAGGGAGACT GTG-3' (restriction sites for EcoRI and NotI have been underlined).

badA from Rhodopseudomonas palustris (GenBank: L42322.1) and pqsA from Pseudomonas aeruginosa (NdeI/XhoI) had been previously cloned 21. badA was first cloned into pCDF-duet1 (NdeI/KpnI) and then ACS 
was subcloned into pCDF-duet1 containing badA (pCRgACS-badA) (BamHI/NotI). Subsequently, RgACSbadA was subcloned into pET-duet1 and pRSF-duet1 (BamHI/EcoRV) using polymerase chain reaction (PCR). Two primers $5^{\prime}$-ATGGATCCGATGGAATCCCTGAA GGAGATG-3 ${ }^{\prime}$ and $5^{\prime}$-GCGGCCGCTCAGCCCAACAC ACCCTCG-3' were used (restriction sites for BamHI and Not $\mathrm{I}$ have been underlined). $p q s A$ was first cloned into pCDF-duet1 (NdeI/XhoI) and ACS was subcloned into pCDF-duet1 containing pqsA (pC-RgACS-pqsA) (BamHI/NotI) and then RgACS-pqsA was subcloned into pET-duet1 (pE-RgACS-pqsA) (BamHI/XhoI).

pA-accABCD encoding acetyl-CoA carboxylase had been cloned previously [32]. TrpE was cloned as described in Lee et al. [20] and subcloned into pCDFduet1 (pC-trpE) (BamHI:BglII/XhoI). aroG and aroG ${ }^{\mathrm{f}}-$ both of which had been previously cloned [18] - were subcloned into pC-trpE (EcoRI/NotI). pC-aroL-aroEaroD-aroB-aroG $\mathrm{f}^{\mathrm{f}}$-ppsA-tktA-trpE had been constructed previously [21]. The E. coli pyruvate dehydrogenase complex variant (PDHm) was cloned as described in Bocanegra et al. [33]. ackA encoding acetate kinase (AAC75356) and pta encoding phosphate acetyltransferase (AAC75357) were cloned from E. coli using PCR. pta was subcloned into pACYC-duetI (NdeI/XhoI), and ackA was subcloned into the resulting plasmid (EcoRI/HindIII) to give rise to pA-ack-pta. acs-encoding E. coli acetylcoenzyme A synthetase (BAE78071)-was subcloned into pACYC-duetI (EcoRI/HindIII) (pA-acs). ACC encoding acetyl-CoA carboxylase from Photorhabdus luminescens (accABCD) had been cloned previously [21].

NMT from Ruta graveolens had been cloned previously [20]. In order to prepare the pC-aroG-NMT-trpE construct, $\operatorname{trp} E$ was amplified using a forward primer containing a BamHI site and a reverse primer containing a XhoI site, and was subcloned into pCDF-duet1 (BglII/BamHI) (pC-trpE). NMT was amplified with a forward primer containing a BamHI site, and a reverse primer containing an $A f l \mathrm{II}$ site, following which it was subcloned into pC-trpE (BamHI/AflII). The resulting construct was digested with $B a m H I / X h o I$, and was then subcloned into pCDF-duet1 (BglII/XhoI) (pCNMT-trpE). aroG or aroG ${ }^{\mathrm{f}}$ were amplified using primers containing EcoRI (forward primer) and NotI sites (reverse primer) and were subcloned into pC-NMT-trpE $(E c o R \mathrm{I} / N o t \mathrm{I})$. The constructs and the strains used in this study were listed in Table 1.

\section{Production and analysis of DHA and NMA in E. coli}

The overnight cultures of $E$. coli transformants were inoculated into a fresh LB containing appropriate antibiotics and growth at $37{ }^{\circ} \mathrm{C}$ until $\mathrm{OD}_{600}=1$. Cells were harvested and resuspended in M9 medium containing $2 \%$ glucose, $1 \%$ yeast extract, antibiotics, $1 \mathrm{mM}$ isopropyl $\beta$-D-1-thiogalactopyranoside (IPTG) in a test tube except that the synthesis of NMA and DHA was monitored for $27 \mathrm{~h}$ in a flask. The cells were grown at $30{ }^{\circ} \mathrm{C}$ with shaking for $24 \mathrm{~h}$. The culture supernatant was extracted with three volumes of ethyl acetate (EA). The upper layer-after centrifugation-was collected and dried. The dried sample was dissolved in $60 \mu \mathrm{L}$ dimethyl sulfoxide (DMSO).

To analyze the formation of DHA and NMA, Thermos Ultimate 3000 HPLC (high performance liquid chromatography) equipped with a photodiode array (PDA) detector and a Varian C18 reversed-phase column (Varian, $4.60 \times 250 \mathrm{~mm}, 3.5 \mu \mathrm{m}$ particle size) was used [21].

The synthesized DHA was purified using HPLC. The mobile phase consisted of water and acetonitrile (7:3, $\mathrm{v} / \mathrm{v})$, and no gradient was applied. The structure of the purified compounds was determined using proton nuclear resonance spectroscopy (NMR). DHA (1,3-dihydroxy-9(10H)-acridone), ${ }^{1} \mathrm{H}$ NMR (500 MHz, DMSO$\left.d_{6}\right): \delta 6.00(\mathrm{~d}, J=2.0 \mathrm{~Hz}, \mathrm{H}-2), 6.30(\mathrm{~d}, J=2.0 \mathrm{~Hz}, \mathrm{H}-4)$, 7.25 (ddd, $J=8.3,7.0,1.0 \mathrm{~Hz}, \mathrm{H}-7$ ), 7.47 (dd, $J=8.3$, $1.4 \mathrm{~Hz}, \mathrm{H}-8$ ), 7.72 (ddd, $J=8.2,7.0,1.4, \mathrm{H}-6$ ), 8.15 (dd, $J=8.2,1.0 \mathrm{~Hz}, \mathrm{H}-5)$. To determine the structure of NMA (1,3-dihydroxy-10-methylacridone), thin layer chromatography (TLC, silica gel 60 F254, Millipore) was used to purify the putative NMA. Ethyl acetate and hexane $(2: 1(\mathrm{v} / \mathrm{v}))$ were used as developing solvents. The purified sample was dissolved in acetone- $d_{6}$. The chemical shifts for ${ }^{1} \mathrm{H}$ and ${ }^{13} \mathrm{C}$ NMR data were referenced to that of tetramethylsilane (TMS). In order to verify the structure, COSY, TOCSY, NOESY, ${ }^{1} \mathrm{H}_{-}{ }^{13} \mathrm{C}$ HMQC, and ${ }^{1} \mathrm{H}-$ ${ }^{13} \mathrm{C}$ HMBC were used. The mixing time for TOCSY and NOESY was $60 \mathrm{~ms}$ and $1 \mathrm{~s}$, respectively. The delay in the evolution of long-ranged couplings was $70 \mathrm{~ms}$ in HMBC. ${ }^{1} \mathrm{H}$ NMR $\left(500 \mathrm{MHz}\right.$, acetone- $\left.d_{6}\right): \delta 6.19(\mathrm{~d}, J=1.9 \mathrm{~Hz}$, $\mathrm{H}-2), 6.50$ (d, $J=1.9 \mathrm{~Hz}, \mathrm{H}-4), 7.78$ (dd, $J=8.8,1.2 \mathrm{~Hz}$, $\mathrm{H}-5$ ), 7.79 (m, H-6), 7.34 (m, H-7), 8.40 (dd, J=8.0, $1.4 \mathrm{~Hz}, \mathrm{H}-8), 3.90\left(\mathrm{~s}, \mathrm{~N}-\mathrm{CH}_{3}\right) .{ }^{13} \mathrm{C} \mathrm{NMR}(500 \mathrm{MHz}$, acetone- $\left.d_{6}\right): \delta 95.26(\mathrm{C}-2), 90.23(\mathrm{C}-4), 114.7(\mathrm{C}-5), 133.4(\mathrm{C}-$ 6), $120.6(\mathrm{C}-7), 125.4(\mathrm{C}-8), 179.9(\mathrm{C}-9), 33.03\left(\mathrm{~N}-\mathrm{CH}_{3}\right)$. In 
Table 1 Plasmids and strains used in the present study

\begin{tabular}{|c|c|c|}
\hline Plasmids or E. coli strain & Relevant properties or genetic marker & Source or reference \\
\hline \multicolumn{3}{|l|}{ Plasmids } \\
\hline pACYCDuet-1 & P15A ori, $\mathrm{Cm}^{r}$ & Novagen \\
\hline pCDFDuet-1 & CloDE13 ori, Str ${ }^{r}$ & Novagen \\
\hline pETDuet-1 & f1 ori, Ampr & Novagen \\
\hline pRSFDuet-1 & RSF ori, Kana' & Novagen \\
\hline pC-CmACS-badA & pCDFDuet + ACS from Citrus microcarpa + badA from Rhodopseudomonas palustris & This study \\
\hline pC-RgACS-badA & pCDFDuet + ACS from Ruta graveolens + badA from R.palustris & This study \\
\hline pC-RgACS-pqsA & pCDFDuet + ACS from Ruta graveolens + pqs A from Pseudomonas aeruginosa & This study \\
\hline $\mathrm{pC}-\mathrm{CmACS}-\mathrm{pqs} A$ & pCDFDuet + ACS from C. microcarpa + pqsA from P. aeruginosa & This study \\
\hline pE-RgACS-badA & pETDuet + ACS from Ruta graveolens + badA from R. palustris & This study \\
\hline pE-RgACS-pqsA & pETDuet + ACS from Ruta graveolens + pqsA from $P$. aeruginosa & This study \\
\hline pR-RgACS-badA & pRSFDuet + ACS from Ruta graveolens + badA from R. palustris & This study \\
\hline pA-accABCD & pACYCDuet + accABCD from Photorhabdus luminescens & Kim et al. [32] \\
\hline pA-Acs & pACYCDuet + Acs from Escherichia coli & This study \\
\hline pA-ackA-pta & pACYCDuet + ackA-pta from E. coli & This study \\
\hline $\mathrm{pA}-\mathrm{PDHm}$ & pACYCDuet + PDHm from E. coli & This study \\
\hline pC-trpE & pCDFDuet + trpE from E. coli & This study \\
\hline pC-aroG-trpE & $\begin{array}{l}\text { pCDFDuet + aroG from Escherichia coli in the first multiple cloning site (MCS1) + trpE from E. coli in the } \\
\text { second MCS (MCS2) }\end{array}$ & This study \\
\hline pC-aroGf-trpE & pCDFDuet + aroG from E. coli in MCS1 + trpE in MCS2 & This study \\
\hline pC-aroG-ppsA-tktA-trpE & pCDFDuet $+\operatorname{aroG}$, , ppsA and tktA from E. coli in MCS1 + trpE in MCS2 & This study \\
\hline pC-aroL-aroGf-ppsA-tktA-trpE & pCDFDuet + aroL, aroGf, ppsA and tktA from E. coli in MCS1 + trpE in MCS2 & This study \\
\hline $\begin{array}{l}\text { pC-aroL-aroE-aroD-aroB-aroG }{ }^{f}- \\
\text { ppsA-tktA-trpE }\end{array}$ & pCDFDuet + aroL, aroE, aroD, aroB, aroG ${ }^{f}, p p s A$ and tktA from E. coli in MCS1 + trpE in MCS2 & This study \\
\hline pC-NMT & pCDFDuet + NMT from Ruta graveolens & This study \\
\hline pC-NMT-trpE & pCDFDuet + NMT from R. gravealens + trpE from E. coli & This study \\
\hline pC-aroG-NMT-trpE & pCDFDuet + aroG from E. coli in MCS1 + NMT and trpE in MCS2 & This study \\
\hline pC-aroG-NMT-trpE & pCDFDuet $+\operatorname{aroG}^{f}$ from E. coli in MCS1 + NMT and trpE in MCS2 & This study \\
\hline \multicolumn{3}{|l|}{ Strains } \\
\hline $\mathrm{DH} 5 \mathrm{a}$ & F- 980 lacZ $\triangle \mathrm{M} 15 \Delta$ (lacZYA-argF)U169 recA1 endA1 hsdR17(rK-, mK +) phoA supE44 $\lambda$ - thi-1 gyrA96 relA1 & Novagen \\
\hline BL21 (DE3) & $\mathrm{F}^{-} \operatorname{ompThsdS}_{B}\left(\mathrm{r}_{\mathrm{B}}^{-} \mathrm{m}_{\mathrm{B}}^{-}\right)$gal dcm lon (DE3) & Novagen \\
\hline B-DHA1 & E. coli BL21 (DE3) harboring pC-RgACS-BadA & This study \\
\hline B-DHA2 & E. coli BL21 (DE3) harboring pC-CmACS-BadA & This study \\
\hline B-DHA3 & E. coli BL21 (DE3) harboring pE-RgACS-BadA, pA-PlaccABCD, pC-TrpE & This study \\
\hline B-DHA4 & E. coli BL21 (DE3) harboring pE-RgACS-BadA, pA-PlaccABCD, pC-aroG-TrpE & This study \\
\hline B-DHA5 & E. coli BL21 (DE3) harboring pE-RgACS-BadA, pA-PlaccABCD, pC-aroG-TrpE & This study \\
\hline B-DHA6 & E. coli BL21 (DE3) harboring pE-RgACS-BadA, pA-PlaccABCD, pC-aroL-aroE-aroD-aroB-aroG-ppsA-tktA-trpE & This study \\
\hline B-DHA7 & E. coli BL21 (DE3) harboring pR-RgACS-BadA, pA-PlaccABCD, pC-TrpE & This study \\
\hline B-DHA8 & E. coli BL21 (DE3) harboring pR-RgACS-BadA, pA-PlaccABCD, pC-aroG-trpE & This study \\
\hline B-DHA9 & E. coli BL21 (DE3) harboring pR-RgACS-BadA, pA-PlaccABCD, pC-aroG-trpE & This study \\
\hline B-DHA10 & E. coli BL21 (DE3) harboring pR-RgACS-BadA, pA-PlaccABCD, pC-aroL-aroE-aroD-aroB-aroG-ppsA-tktA-trpE & This study \\
\hline B-NMA1 & E. coli BL21 (DE3) harboring pC-RgACS-PqsA & This study \\
\hline B-NMA2 & E. coli BL21 (DE3) harboring pC-CmACS-PqsA & This study \\
\hline B-NMA3 & E. coli BL21 (DE3) harboring pE-RgACS-PqsA, pC-NMT-TrpE, pA-PlaccABCD & This study \\
\hline B-NMA4 & E. coli BL21 (DE3) harboring pE-RgACS-PqsA, pC-NMT-TrpE, pA-ack-pta & This study \\
\hline B-NMA5 & E. coli BL21 (DE3) harboring pE-RgACS-PqsA, pC-NMT-TrpE, pA-mPDH & This study \\
\hline B-NMA6 & E. coli BL21 (DE3) harboring pE-RgACS-PqsA, pC-NMT-TrpE, pA-acs & This study \\
\hline B-NMA7 & E. coli BL21 (DE3) harboring pE-RgACS-PqsA, pC-NMT-TrpE, pACYCD & This study \\
\hline B-NMA8 & E. coli BL21 (DE3) harboring pE-RgACS-PqsA, pC-aroG-NMT-TrpE, pA-PlaccABCD & This study \\
\hline B-NMA9 & E. coli BL21 (DE3) harboring pE-RgACS-PqsA, pC-aorGf-NMT-TrpE, pA-PlaccABCD & This study \\
\hline
\end{tabular}


the ${ }^{1} \mathrm{H}$ spectrum, six peaks were observed in the aromatic region, while a single peak was observed at $3.90 \mathrm{ppm}$. All peaks in the aromatic region were assigned using COSY and TOCSY. The $\mathrm{N}$-attached methyl group was thought to be responsible for the peak at $3.899 \mathrm{ppm}$ as it showed two cross-peaks with $\mathrm{H}-5$ and $\mathrm{H}-4$ in NOESY.

\begin{abstract}
Abbreviations
ACC: Acetyl-coenzyme A carboxylase; ackA: Acetate kinase; ANMT: Anthranilate $\mathrm{N}$-methyltransferase; ASC: Acridone synthase; CoA: Coenzyme A; DHA: 1,3-Dihydroxy-9(10H)-acridone; DMSO: Dimethyl sulfoxide; HPLC: High performance liquid chromatography; IPTG: Isopropyl $\beta$-D-1-thiogalactopyranoside; NMA: 1,3-Dihydroxy-10-methylacridone; NMR: Nuclear magnetic resonance spectroscopy; NMT: N-methyltransferase gene; PDA: Photodiode array; PDHm: Pyruvate dehydrogenase complex variant; PKS: Polyketide synthases; pta: Phosphate acetyltransferase.
\end{abstract}

\section{Acknowledgements}

Not applicable.

\section{Authors' contributions}

GSC, BGK and JHA designed experiments. GSC, HJC BGK, and JHA performed the experiments and analyzed the data. GSC, HJC and JHA wrote the manuscript. All authors read and approved the final manuscript.

\section{Funding}

This work was supported by grants from the National Research Foundation (NRF-2019R1A2C1002714) funded by the Ministry of Education, Science and Technology, and from the Next-Generation BioGreen 21 Program (PJ01326001), Rural Development Administration, Republic of Korea.

\section{Availability of data and materials}

All data generated during this study are included in this published article.

\section{Ethics approval and consent to participate}

This manuscript does not report data collected from humans or animals.

\section{Consent for publication}

This manuscript does not contain any individual persons' data.

\section{Competing interests}

The authors declare that they have no competing interests.

\section{Author details}

${ }^{1}$ Department of Bioscience and Biotechnology, Bio/Molecular Informatics Center, Konkuk University, Seoul 05029, Republic of Korea. ${ }^{2}$ Department of Forest Resources, Gyeongnam National University of Science and Technology, 33 Dongjin-ro, Jinju-si, Gyeongsangman-do 52725, Republic of Korea.

Received: 27 January 2020 Accepted: 13 March 2020

Published online: 20 March 2020

\section{References}

1. Harjo B, Wibowo C, Ng KM. Development of natural product manufacturing processes: phytochemicals. Chem Eng Res Des. 2004;82:1010-28.

2. Hartmann T. From waste products to ecochemicals: fifty years research of plant secondary metabolism. Phytochemistry. 2007;68:22-4.

3. Heba A-EE-G, Farghaly AO, Mohammed AH. Acridone-based acetylcholinesterase inhibitors; synthesis, antioxidant activity and molecular modeling. Int J Drug Deliv. 2018;10:25-35.

4. Kuzovkina IN, Kislov LD, Zhivopistseva MN, Rozsa Z, Szendrei K. Acridone alkaloids of callus tissue of Ruta graveolens. Chem Nat Compd. 1984;20:716-9.

5. Bernardino AMR, Azevedo AR, Pinheiro LCS, Rodrigues CR, Castro HC, Frugulhetti ICPP, Albuquerque MG. SAR of a series of anti-HSV-1 acridone derivatives, and a rational acridone-based design of a new anti-HSV-1 $3 \mathrm{H}$-benzo[b]pyrazolo[3,4-h]-1,6-naphthyridine series. Bioorg Med Chem. 2008:16:313-21.

6. Goodell JR, Madhok AA, Hiasa H, Ferguson DM. Synthesis and evaluation of acridine- and acridone-based anti-herpes agents with topoisomerase activity. Bioorg Med Chem. 2006;14:5467-80.

7. Michael JP. Quinoline, quinazoline and acridone alkaloids. Nat Prod Rep. 2008;25:166-87.

8. Putic A, Stecher L, Prinz H, Müller K. Structure-activity relationship studies of acridones as potential antipsoriatic agents. 2. Synthesis and antiproliferative activity of 10-substituted hydroxy-10H-acridin-9-ones against human keratinocyte growth. Eur J Med Chem. 2010;45:5345-52.

9. Schmidt A, Liu M. Recent advances in the chemistry of acridines. Adv Heterocycl Chem. 2015;115:287-353.

10. Baumert A, Porzel A, Schmidt A, Gröger D. Formation of 1,3-dihydroxy$\mathrm{N}$-methylacridone from $\mathrm{N}$-methylanthraniloyl-CoA and malonyl-CoA by cell-free extracts of Ruta graveolens. Z Naturforsch. 1992;47:365-8.

11. Lukačin R, Springob K, Urbanke C, Ernwein C, Schroder G, Schroder J, Matern U. Native acridone synthases I and II from Ruta graveolens L. form homodimers. FEBS Lett. 1999;1999(448):135-40.

12. Rohde B, Hans J, Martens S, Baumert A, Hunziker P, Matern U. Anthranilate $\mathrm{N}$-methyltransferase, a branch-point enzyme of acridone biosynthesis. Plant J. 2008;53:541-53.

13. Wanibuchi K, Zhang P, Abe T, Noguchi H, Abe I, Chen G, Kohno T. An acridone-producing novel multifunctional type III polyketide synthase from Huperzia serrate. FEBS J. 2007;274:1073-82.

14. Mori T, Shimokawa Y, Matsui T, Kinjo K, Kato R, Noguchi H, Abe I. Cloning and structure-function analyses of quinolone- and acridoneproducing novel type III polyketide synthases from Citrus microcarpa. J Biol Chem. 2013;288:28845-58.

15. Jing $D$, Zengyi $S$, Huimin Z. Engineering microbial factories for synthesis of value-added products. J Ind Microb Biotechnol. 2011;38:873-90.

16. Jiang $M$, Zhang $H$. Engineering the shikimate pathway for biosynthesis of molecules with pharmaceutical activities in E. coli. Curr Opin Biotechnol. 2016;42:1-6.

17. Knaggs AR. The biosynthesis of shikimate metabolites. Nat Prod Rep. 2003;20:119-36.

18. Kim M-J, Kim B-G, Ahn J-H. Biosynthesis of bioactive O-methylated flavonoids in Escherichia coli. Appl Microbiol Biotechnol. 2013;97:7195-204.

19. Lee SJ, Sim G-Y, Lee Y, Kim B-G, Ahn J-H. Engineering of Escherichia coli for the synthesis of $\mathrm{N}$-hydroxycinnamoyl tryptamine and serotonin. J Ind Microb Biotechnol. 2017;44:1551-60.

20. Lee HL, Kim S-Y, Kim EJ, Han DY, Kim B-G, Ahn J-H. Synthesis of methylated anthranilate derivatives using engineered strains of Escherichia coli. J Microb Biotechnol. 2019:29:839-44.

21. Choo HJ, Ahn J-H. Synthesis of three bioactive aromatic compounds by introducing polyketide synthase genes into engineered Escherichia coli. J Agric Food Chem. 2019;67:8581-9.

22. Springob K, Lukacin R, Ernwein C, Groning I, Matern U. Specificities of functionally expressed chalcone and acridone synthases from Ruta graveolens. Eur J Biochem. 2000;267:6552-9.

23. Cardenas J, Da Silva NA. Engineering cofactor and transport mechanisms in Saccharomyces cerevisiae for enhanced acetyl-CoA and polyketide biosynthesis. Metab Eng. 2016;36:80-9.

24. Leonard E, Lim K-H, Saw P-N, Koffas MAG. Engineering central metabolic pathways for high-level flavonoid production in Escherichia coli. Appl Environ Microbiol. 2007;73:3877-86

25. Na D, Yoo SM, Chung H, Park H, Park JH, Lee SY. Metabolic engineering of Escherichia coli using synthetic small regulatory RNAs. Nat Biotechnol. 2013;31:170-4.

26. Wu J, Du G, Chen J, Zhou J. Enhancing flavonoid production by systematically tuning the central metabolic pathways based on a CRISPR interference system in Escherichia coli. Sci Rep. 2015;5:13477.

27. Lim CG, Fowler ZL, Hueller T, Schaffer S, Koffas MAG. High-yield resveratrol production in engineered Escherichia coli. Appl Environ Microbiol. 2011;77:3451-60

28. Baumert A, Maier W, Gröger D, Deutzmann R. Purification and properties of acridone synthase from cell suspension cultures of Ruta graveolens L. Z Naturforsch. 1994;49:26-32. 
29. De Mey M, De Maeseneire S, Soetaert W, Vandamme E. Minimizing acetate formation in E. coli fermentations. J Ind Microb Biotechnol. 2007;34:689-700

30. Enjalbert B, Millard P, Dinclaux M, Portais J-C, Létisse F. Acetate fluxes in Escherichia coli are determined by the thermodynamic control of the PtaAckA pathway. Sci Rep. 2017;7:42135.

31. Xu P, Ranganathan S, Fowler Z, Maranas CD, Koffas MAG. Genomescale metabolic network modeling results in minimal interventions that cooperatively force carbon fluxtowards malonyl-CoA. Metab Eng. 2011;13:578-87.
32. Kim BG, Lee HJ, Ahn J-H. Biosynthesis of pinocembrin from glucose using engineered Escherichia coli. J Microb Biotechnol. 2014;24:1536-41.

33. Bocanegra JA, Scrutton NS, Perham RN. Creation of an NADP-dependent pyruvate dehydrogenase multienzyme complex by protein engineering. Biochemistry. 1993;32:2737-40.

\section{Publisher's Note}

Springer Nature remains neutral with regard to jurisdictional claims in published maps and institutional affiliations.
Ready to submit your research? Choose BMC and benefit from:

- fast, convenient online submission

- thorough peer review by experienced researchers in your field

- rapid publication on acceptance

- support for research data, including large and complex data types

- gold Open Access which fosters wider collaboration and increased citations

- maximum visibility for your research: over 100M website views per year

At BMC, research is always in progress.

Learn more biomedcentral.com/submissions 